\title{
Changes of Metabolites in Acute Ischemic Stroke and Its Subtypes
}

\begin{abstract}
Xin Wang 1,2, Luyang Zhang ${ }^{1,2}$, Wenxian Sun 1,2, Lu-lu Pei ${ }^{1,2}$, Mengke Tian 1,2, Jing Liang ${ }^{1,2}$, Xinjing Liu ${ }^{1,2}$, Rui Zhang ${ }^{1,2}$, Hui Fang ${ }^{1,2}$, Jun $W_{u^{1,2}}$, Shilei Sun ${ }^{1,2}$, Yuming $X_{u^{1,2 *}}$, Jian-Sheng Kang ${ }^{1 *}$ and Bo Song ${ }^{1,2 *}$

${ }^{1}$ Department of Neurology, The First Affiliated Hospital of Zhengzhou University, Zhengzhou, China, ${ }^{2}$ Henan Key Laboratory of Cerebrovascular Diseases, Zhengzhou, China
\end{abstract}

Existing techniques have many limitations in the diagnosis and classification of ischemic stroke (IS). Considering this, we used metabolomics to screen for potential biomarkers of IS and its subtypes and to explore the underlying related pathophysiological mechanisms. Serum samples from 99 patients with acute ischemic stroke (AIS) [the AIS subtypes included 49 patients with large artery atherosclerosis (LAA) and 50 patients with small artery occlusion (SAO)] and 50 matched healthy controls ( $\mathrm{HCs}$ ) were analyzed by non-targeted metabolomics based on liquid chromatography-mass spectrometry. A multivariate statistical analysis was performed to identify potential biomarkers. There were 18 significantly different metabolites, such as oleic acid, linoleic acid, arachidonic acid, L-glutamine, L-arginine, and L-proline, between patients with AIS and HCs. These different metabolites are closely related to many metabolic pathways, such as fatty acid metabolism and amino acid metabolism. There were also differences in metabolic profiling between the LAA and SAO groups. There were eight different metabolites, including L-pipecolic acid, 1-Methylhistidine, PE, LysoPE, and LysoPC, which affected glycerophospholipid metabolism, glycosylphosphatidylinositolanchor biosynthesis, histidine metabolism, and lysine degradation. Our study effectively identified the metabolic profiles of IS and its subtypes. The different metabolites between LAA and SAO may be potential biomarkers in the context of clinical diagnosis. These results highlight the potential of metabolomics to reveal new pathways for IS subtypes and provide a new avenue to explore the pathophysiological mechanisms underlying IS and its subtypes.

Keywords: ischemic stroke, metabolites, non-targeted metabolomics, TOAST, biomarkers

\section{INTRODUCTION}

Stroke is one of the main causes of human death and disability (Wang et al., 2014) and is associated with a high rate of disability and recurrence. According to population-based studies (Benjamin et al., 2017), ischemic stroke (IS) accounts for more than $80 \%$ of all strokes. According to its etiology and imaging, IS can be categorized into five subtypes (Adams et al., 1993; Chen et al., 2012), including large artery atherosclerosis (LAA), small artery occlusion (SAO), cardioembolism, stroke of other determined cause, and stroke of undetermined cause. The classification of IS can help with the early treatment and prevention of long-term recurrence in patients (Montaner et al., 2008). However, the diagnosis and classification of IS mainly rely on neuroimaging techniques, 
which are scarce, expensive, and time-consuming (Latchaw et al., 2009). Therefore, new biomarkers for the rapid and accurate prediction, diagnosis, and classification of IS might play a positive role in clarifying the pathophysiological mechanism of IS and promoting the secondary prevention and management of patients with IS.

It is difficult to release macromolecules from the brain into the blood due to the presence of the blood-brain barrier (Jickling and Sharp, 2015). Some conventional detection methods make it difficult to detect specific sensitive different metabolites in patients with IS. However, with the development of the emerging science of metabolomics, it may be possible to identify specific small molecular biomarkers in patients with IS and determine the underlying etiology. Metabolomics is an effective method to reveal biomolecules' phenotypes. This method enables the identification of changes in small molecular metabolites in various diseases, which can greatly help in understanding and diagnosing diseases. Many studies using metabolomics have revealed the differences in metabolites between patients with acute ischemic stroke (AIS) and healthy controls (HCs) (Jung et al., 2011; Kimberly et al., 2013). To date, few studies have explored the differences in metabolites between the LAA and SAO subtypes of IS. In this study, non-targeted metabolites based on liquid chromatography-mass spectrometry (LC-MS) were used to study the different metabolites between patients with AIS and the HCs and between patients with the LAA and SAO subtypes of IS. The proposed method offers important advantages over traditional alternatives, ensuring that it is feasible to screen potential biomarkers and further explore the relevant underlying pathophysiological mechanisms.

\section{MATERIALS AND METHODS}

\section{Study Population}

In this study, 99 patients with AIS within 7 days of onset were included in the AIS group, including 49 patients with LAA and 50 patients with SAO. Among the 99 AIS patients, the time from onset to blood withdrawal was within $24 \mathrm{~h}$ among 45 patients and within $72 \mathrm{~h}$ among 38 patients. The remaining 16 patients showed transient ischemic attack symptoms within 7 days, but the blood samples were only collected at around $72 \mathrm{~h}$ after the symptoms begin to persist. A total of $50 \mathrm{HCs}$ with age, sex, and risk factors matched with the AIS group were recruited. All patients needed to meet the following conditions: (1) no history of stroke or coronary heart disease, (2) no history of malignant tumor or autoimmune disease, (3) blood samples can be obtained within $24 \mathrm{~h}$ of enrollment, and (4) head magnetic resonance imaging and angiography were completed during hospitalization. The patients in the AIS group, who were hospitalized in the Department of Neurology from October 2015 to December 2016, and the samples of the AIS group were acquired from the ischemic cerebrovascular disease database and blood database of The First Affiliated Hospital of Zhengzhou University. The details of the database and related articles have been published elsewhere (Song et al., 2013; Kelly et al., 2016; Wang et al., 2020). All patients with AIS were diagnosed according to the diagnostic criteria of the World Health Organization [WHO] (1989). The TOAST classification was evaluated back to back by two professional neurologists. Written informed consent was obtained from all participants or their representatives.

\section{Serum Sample Preparation}

Blood samples of patients with AIS were collected within $24 \mathrm{~h}$ after admission; when collecting, it was ensured that the patients have fasted for at least $8 \mathrm{~h}$. The serum was centrifuged and extracted within $1 \mathrm{~h}$ and refrigerated at $-80^{\circ} \mathrm{C}$. To separate metabolites with different polarities, the same sample underwent two different treatment methods. After melting in ice at $4^{\circ} \mathrm{C}$ for $30-60 \mathrm{~min}, 40 \mu \mathrm{l}$ of serum was taken into a 1.5-ml centrifuge tube for a reversed-phase ultra-performance liquid chromatographic analysis, adding $300 \mu \mathrm{l}$ methanol and $1 \mathrm{ml}$ methyl tert-butyl ether to precipitate the protein for $15 \mathrm{~s}$. The sample was then placed in a centrifuge at $12,000 \mathrm{rpm}$ at a constant temperature of $4^{\circ} \mathrm{C}$ for $10 \mathrm{~min}$, the upper solution $(400 \mu \mathrm{l})$ was then evaporated, and the sample was finally redissolved in $100 \mu \mathrm{l}$ methanol. For the serum analyzed by Hydrop interaction liquid chromatography (HILIC), $50 \mu \mathrm{l}$ plus $150 \mu \mathrm{l}$ acetonitrile was added to the centrifuge tube to precipitate the protein, and $100 \mu \mathrm{l}$ of the upper solution was centrifuged under the above-mentioned conditions to be determined.

\section{Chromatographic Condition}

For the C18 separation, mobile phase A consisted of acetonitrile/water (60/40), and mobile phase B was isopropanol/acetonitrile (90/10); both A and B contained $0.1 \%$ formic acid and $10 \mathrm{mmol} / \mathrm{L}$ ammonium acetate. The column was an HSS T3 column $(2.1 \times 100 \mathrm{~mm}, 1.8 \mu \mathrm{m}$, Waters $)$ operated at $45^{\circ} \mathrm{C}$. The flow rate was $300 \mu \mathrm{l} / \mathrm{min}$, and the injection volume was $1 \mu \mathrm{l}$. For the HILIC separation, mobile phase $\mathrm{A}$ was acetonitrile, and mobile phase $\mathrm{B}$ was water; both $\mathrm{A}$ and $\mathrm{B}$ contained $0.1 \%$ formic acid and $10 \mathrm{mmol} / \mathrm{L}$ ammonium acetate. The column was a BEH amide column $(2.1 \times 100 \mathrm{~mm}, 1.7 \mu \mathrm{m}$, Waters) operated at $40^{\circ} \mathrm{C}$. The flow rate was $300 \mu \mathrm{l} / \mathrm{min}$, and the injection volume was $1 \mu 1$.

\section{LC-MS Detection}

A metabolomics analysis was performed using a Thermo Scientific Q Exactive hybrid quadrupole Orbitrap mass spectrometer equipped with a HESI-II probe. The positive and negative HESI-II spray voltages were 3.7 and $3.5 \mathrm{kV}$, respectively, the heated capillary temperature was $320^{\circ} \mathrm{C}$, the sheath gas pressure was $30 \mathrm{psi}$, the auxiliary gas setting was $10 \mathrm{psi}$, and the heated vaporizer temperature was $300^{\circ} \mathrm{C}$. Both the sheath gas and the auxiliary gas consisted of nitrogen. The collision gas was also nitrogen at a pressure of 1.5 mTorr. The parameters of the full mass scan were as follows: resolution of 70,000, auto gain control target under $1 \times 106$, maximum isolation time of $50 \mathrm{~ms}$, and $\mathrm{m} / \mathrm{z}$ range of $50-1,500$. The LC-MS system was controlled using Xcalibur 2.2 SP1.48 software (Thermo Fisher Scientific), and data were collected and processed using the same software. 


\section{Untargeted Metabolome Data Processing}

All data obtained from the four assays in the two systems in both positive and negative ion modes were processed using Progenesis QI data analysis software (Non-linear Dynamics, Newcastle, United Kingdom) to impute raw data, peak alignment, picking, and normalization to produce peak intensities for retention time $\left(t_{R}\right)$ and $\mathrm{m} / \mathrm{z}$ data pairs. The ranges of automatic peak picking for the $\mathrm{C} 18$ were between 1 and $16 \mathrm{~min}$ and between 1 and $12 \mathrm{~min}$, respectively. Next, the adduct ions of each "feature" $\left(\mathrm{m} / \mathrm{z}, t_{R}\right)$ were deconvoluted, and these features were identified in the human metabolome database (HMDB) and Lipidmaps.

To monitor a system's stability and performance and the reproducibility of the sample, quality control (QC) samples were prepared by pooling equal volumes of each serum sample. The pretreatment of serum QC samples was performed in accordance with real samples. For repeatable metabolic analyses, three features of the analytical system must be stable: (1) retention time, (2) signal intensity, and (3) mass accuracy. In this study, three QCs were continuously injected at the beginning of the run. QC samples are then injected at regular intervals of six or eight samples throughout the analytical run to provide data from which repeatability can be assessed.

The features were selected based on their coefficients of variation (CVs) with QC samples; features with CVs over 15\% were eliminated.

\section{Statistical Analysis}

The classified variables and continuous variables in the baseline information on participants were compared by $\chi^{2}$ test and $t$-test in SPSS, respectively. Data are presented as mean \pm SD or the percentage, as appropriate. A multivariate statistical analysis was performed using principal component analysis (PCA) and orthogonal projections to latent structures-discriminant analysis (OPLS-DA) multivariate statistical methods in SIMCA (14.1) software. In this study, the variable importance in the projection (VIP) value of the OPLS-DA model (threshold $>1$ ) and the $P$-value of $t$-test $(P<0.05)$ were used to identify the different metabolites. The qualitative method of different metabolites consists of searching in HMDB (to compare the $\mathrm{m} / \mathrm{z}$ or molecular mass, error limit $0.01 \mathrm{Da}$ ). The OPLS-DA model was then validated by permutation tests. A pathway analysis was performed using MetaboAnalyst 4.0.

\section{RESULTS}

\section{Baseline Characteristics}

In this study, there were 99 people in the AIS group and 50 people in the control group. The baseline characteristics are shown in Table 1. There were 73 males and 26 females with an average age of 58.06 years in the AIS group and 36 men and 14 women with an average age of 57.60 years in the control group. We found no significant difference in age, sex, hyperlipidemia, and diabetes mellitus between the AIS and control groups $(P>0.05)$.

\section{PCA and OPLS-DA}

The PCA of the unsupervised model was used to analyze the differences and intra-group variation among the LAA, SAO, and HC groups, in which $R^{2} X$ was used to judge the quality of the model, and $Q^{2}$ represented the predictable variables of the model. As shown in Figures 1A-C, there was a slight separation among the three groups on the score plots in both the C18 column and the HILIC column (C18-positive: $R^{2} X=0.789$, $Q^{2}=0.547$; C18-negative: $R^{2} X=0.817, Q^{2}=0.616$; HILIC: $\left.R^{2} X=0.732, Q^{2}=0.453\right)$. To obtain the metabolite information that leads to this difference, supervised models and OPLS-DA were performed. The serum samples in the AIS group and the HC group were separated in the C18 column and HILIC column (C18-positive: $R^{2} Y=0.883, Q^{2}=0.726$; C18-negative: $R^{2} Y=0.964, Q^{2}=0.857$; HILIC: $\left.R^{2} Y=0.985, Q^{2}=0.914\right)$ as were the LAA group and the SAO group (C18-positive: $R^{2} Y=0.916$, $Q^{2}=0.778$; C18-negative: $R^{2} Y=0.909, Q^{2}=0.800$; HILIC: $\left.R^{2} Y=0.953, Q^{2}=0.726\right)$, highlighting the excellence of the models (Figures 1D-I).

\section{Different Metabolites}

The analysis of OPLS-DA in the supervised model is summarized in Table 2. A total of 18 significantly changed metabolites (SCMs)

TABLE 1 | Comparison of baseline characteristics between the patients and the healthy controls.

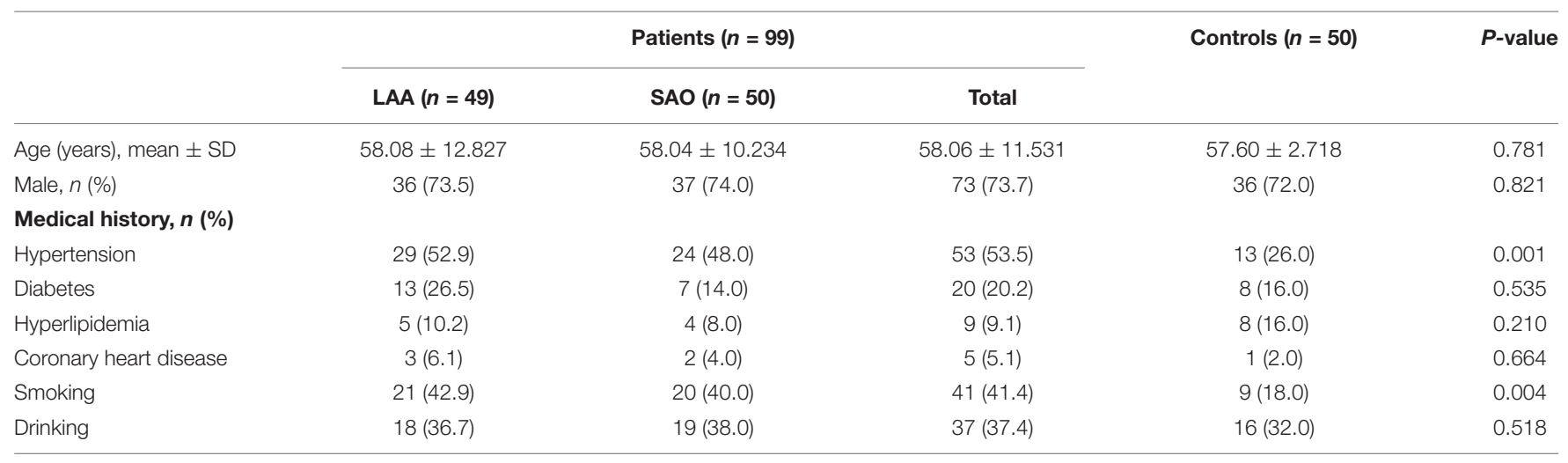

LAA, large artery atherosclerosis; SAO, small artery occlusion. 


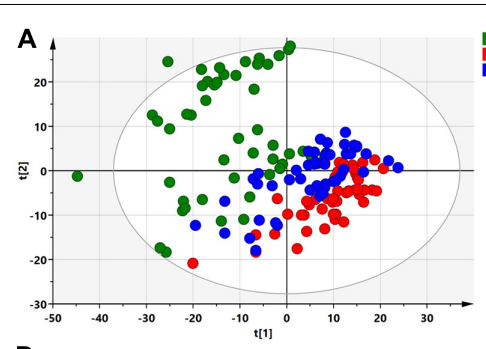

D

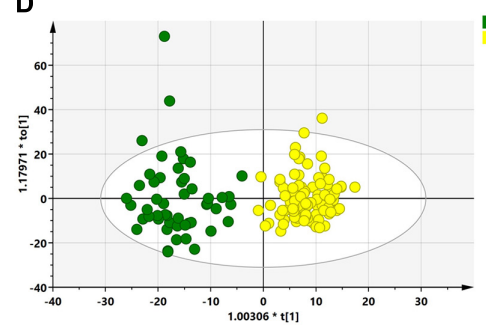

G

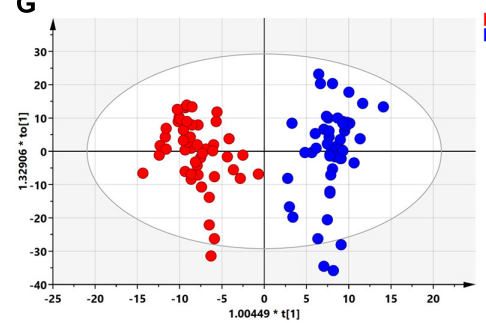

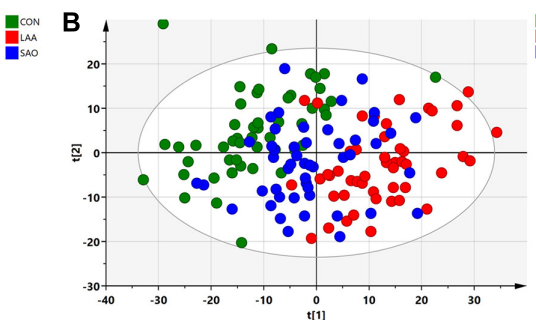

E

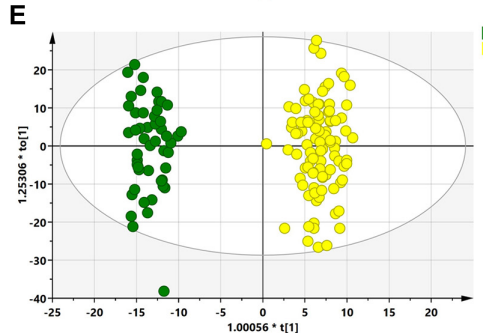

n $_{\text {LAA }}^{\text {LAA }}$

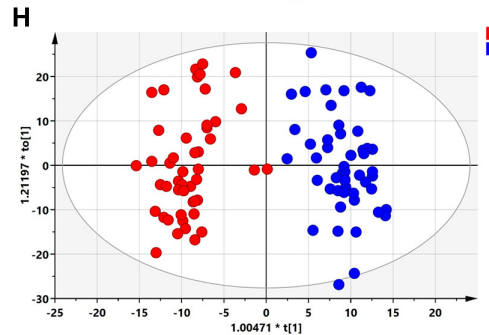

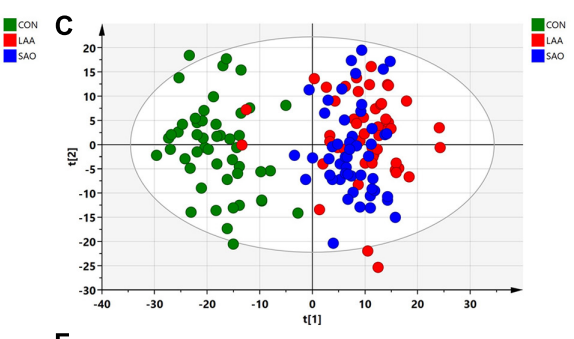

IDON

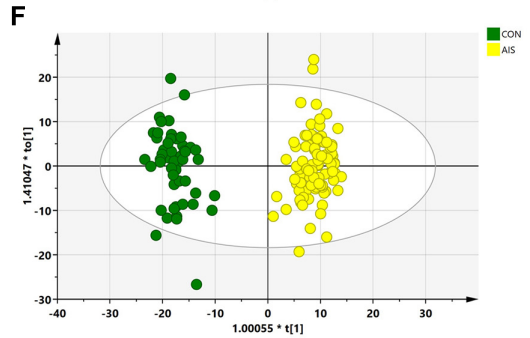

$\ln _{\lim } 1$

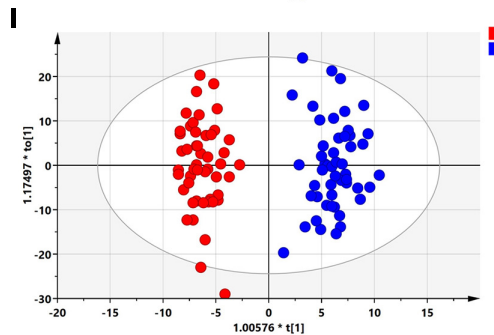

FIGURE 1 | Multivariate statistical analysis of serum metabolic profiling between acute ischemic stroke (AIS) and healthy control (HC) groups. (A) Principal component analysis (PCA) score plots in the C18-positive column $\left(R^{2} X=0.789, Q^{2}=0.547\right)$. (B) PCA score plots in the C18-negative column $\left(R^{2} X=0.817\right.$, $\left.Q^{2}=0.616\right)$. (C) PCA score plots in the Hydrop interaction liquid chromatography (HILIC) column $\left(R^{2} X=0.732, Q^{2}=0.453\right)$. (D) Orthogonal projections to latent structures - discriminant analysis (OPLS-DA) score plots of patients with AIS and HCs in the C18-positive column ( $\left.R^{2} Y=0.883, Q^{2}=0.726\right)$. (E) OPLS-DA score plots of patients with AIS and HCs in the C18-negative column $\left(R^{2} Y=0.964, Q^{2}=0.857\right)$. (F) OPLS-DA score plots of the patients with AIS and HCs in the HILIC column $\left(R^{2} Y=0.985, Q^{2}=0.914\right)$. (G) OPLS-DA score plots of the large artery atherosclerosis (LAA) and the small artery occlusion (SAO) groups in the C18-positive column $\left(R^{2} Y=0.916, Q^{2}=0.778\right)$. (H) OPLS-DA score plots of the LAA and the SAO groups in the C18-negative column $\left(R^{2} Y=0.909, Q^{2}=0.800\right)$. (I) OPLS-DA score plots of the LAA and the SAO groups in the HILIC column $\left(R^{2} Y=0.953, Q^{2}=0.726\right)$.

(VIP $>1, P<0.05$ ) were screened between the AIS group and the $\mathrm{HC}$ group through different chromatographic columns, and a total of eight SCMs were screened between the LAA and SAO groups from the HMDB.

Compared with the HCs, the AIS patients exhibited higher levels of oleic acid, linoleic acid, arachidonic acid (AA), docosahexaenoic acid (DHA), L-palmitoylcarnitine, tetradecanoylcarnitine, dodecanoylcarnitine, and decanoylcarnitine and lower levels of Cer (14:0), Cer (16:0), nonadecanoic acid, 4-hydroxyproline, phosphatidylethanolamine (PE) (18:1), PE (18:0), propionylcarnitine, L-glutamine, L-arginine, and L-proline as shown in the heat map in Figure 2. In comparison to the SAO group, the LAA group was characterized by decreased levels of L-pipecolic acid, 1-methylhistidine, PE (18:2), LysoPE (18:2), LysoPC (18:3), LysoPC (20:0), and LysoPC (18:2) and by increased levels of PE (16:0). Detailed information is shown in Table 2.

\section{Metabolic Pathways}

MetaboAnalyst 4.0 was used to analyze the different metabolic pathways of the groups. The potential different metabolic pathways between the AIS patients and the HCs include linoleic acid metabolism, AA metabolism, arginine and proline metabolism, and alanine, aspartate, and glutamate metabolism. The metabolic pathways of the LAA group and the SAO group probably differ in glycerophospholipid metabolism, glycosylphosphatidylinositol (GPI)-anchor biosynthesis, histidine metabolism, and lysine degradation (Figure 3).

\section{DISCUSSION}

We obtained the serum metabolic profiling of stroke patients by non-targeted metabolomics and discovered that the AIS patients had metabolic disorders. Furthermore, the metabolic profiles of the LAA and SAO subtypes of IS were different. Among the abnormal metabolic indicators, the metabolic disorders of lipids and amino acids are the most obvious. Changes in metabolite patterns lay the foundation for us to further clarify the pathophysiological mechanisms of stroke and find ways to implement innovative clinical diagnoses of stroke classifications.

The brain consumes approximately $20 \%$ of total human energy consumed. Approximately 20\% of the energy consumed 
TABLE 2 | Characteristics of the different metabolites.

\begin{tabular}{|c|c|c|c|c|c|}
\hline Metabolites & Retention time (min) & Mass-to-charge ratio & VIP value & Fold change & $P$-value \\
\hline \multicolumn{6}{|l|}{ Between the AIS and HCs groups } \\
\hline Oleic acid & 5.116 & 281.249 & 2.107 & 2.216 & $<0.00001$ \\
\hline Linoleic acid & 4.465 & 279.233 & 1.844 & 1.970 & 0.00001 \\
\hline Cer (d18:0/14:0) & 8.993 & 512.503 & 1.735 & 0.212 & $<0.00001$ \\
\hline Cer (d18:0/16:0) & 9.546 & 540.534 & 1.732 & 0.213 & $<0.00001$ \\
\hline Arachidonic acid & 4.421 & 303.233 & 1.556 & 1.564 & 0.00030 \\
\hline Non-adecanoic acid & 6.306 & 297.280 & 1.371 & 0.652 & $<0.00001$ \\
\hline Docosahexaenoic acid & 4.207 & 327.233 & 1.306 & 1.342 & 0.00964 \\
\hline 4-Hydroxyproline & 5.939 & 132.066 & 1.270 & 0.520 & $<0.00001$ \\
\hline PE[18:2(9Z,12Z)/18:1(9Z)] & 8.534 & 742.538 & 1.099 & 0.492 & $<0.00001$ \\
\hline PE[18:2(9Z,12Z)/18:0] & 8.989 & 744.553 & 1.099 & 0.514 & $<0.00001$ \\
\hline L-Palmitoylcarnitine & 1.433 & 400.342 & 1.098 & 1.338 & $<0.00001$ \\
\hline Propionylcarnitine & 2.604 & 218.139 & 1.097 & 0.716 & $<0.00001$ \\
\hline Tetradecanoylcarnitine & 1.473 & 372.311 & 1.095 & 1.382 & 0.00015 \\
\hline L-Glutamine & 6.353 & 147.077 & 1.094 & 0.810 & $<0.00001$ \\
\hline L-Arginine & 7.316 & 175.119 & 1.093 & 0.742 & $<0.00001$ \\
\hline Dodecanoylcarnitine & 1.522 & 344.280 & 1.094 & 1.349 & 0.00695 \\
\hline L-Proline & 5.346 & 116.071 & 1.092 & 0.758 & $<0.00001$ \\
\hline Decanoylcarnitine & 1.593 & 316.248 & 1.090 & 1.330 & 0.02110 \\
\hline \multicolumn{6}{|l|}{ Between the LAA and SAO group } \\
\hline L-Pipecolic acid & 4.735 & 130.087 & 1.578 & 1.790 & 0.00065 \\
\hline 1-Methylhistidine & 7.267 & 170.093 & 1.317 & 1.625 & 0.04886 \\
\hline PE [22:6(4Z,7Z,10Z,13Z,16Z,19Z)/16:0] & 8.209 & 764.521 & 1.270 & 0.780 & 0.02704 \\
\hline PE [P-18:0/18:2(9Z,12Z)] & 9.314 & 728.557 & 1.192 & 1.276 & 0.01645 \\
\hline LysoPE [18:2(9Z,12Z)/0:0] & 2.428 & 478.292 & 1.113 & 1.446 & 0.00011 \\
\hline LysoPC [18:3(9Z,12Z,15Z)] & 1.991 & 518.323 & 1.045 & 1.387 & 0.00144 \\
\hline LysoPC (20:0) & 5.009 & 552.402 & 1.033 & 1.254 & 0.00669 \\
\hline LysoPC [18:2(9Z,12Z)] & 2.455 & 520.339 & 1.000 & 1.336 & 0.00013 \\
\hline
\end{tabular}

LAA, large artery atherosclerosis; SAO, small artery occlusion; AIC, acute ischemic stroke; HC, healthy control; PE, phosphatidylethanolamine.

by the brain is provided by the oxidative reaction of fatty acids (Ebert et al., 2003). Neurons are very sensitive to conditions such as ischemia and hypoxia. In order to regulate the lack of energy caused by the AIS, the brain can initiate energy production responses such as fatty acid degradation through negative feedback to maintain homeostasis (Schwartz et al., 2000; Belgardt and Brüning, 2010). Oleic acid and linoleic acid are long-chain fatty acids that can cross the blood-brain barrier to provide energy to the brain (Panov et al., 2014), and L-palmitoylcarnitine is also involved in fatty acid degradation. In this study, compared with the HC group, the increase in oleic acid, linoleic acid, and L-palmitoylcarnitine in the AIS group may be associated with increased fatty acid catabolism in the acute phase of the IS to maintain energy homeostasis. In addition, previous studies have reported that changes in lipid metabolism are associated with mitochondrial dysfunction caused by oxidative stress (Tobe, 2013), which is one of the three main pathophysiological reactions (neurotoxicity, oxidative stress, and inflammation) in IS (Fukuyama et al., 1998; Chamorro et al., 2012; Lai et al., 2014). This result is consistent with the transcriptomic profiling results of IS (Li et al., 2015; Cai et al., 2019). Cai et al. (2019) assessed the patterns of transcriptomic changes at different stages of IS using a mouse model. The results showed that mmu-miR-199a-5p and mmu-miR-199b-3p inhibit the inflammatory response during the recovery phase of IS and exert neuroprotective effects by regulating the Taok1 gene (Cai et al., 2019). This may imply that fatty acid metabolism is related to the regulation of these genes. However, this requires further verification in animal experiments.

Both AA and DHA are polyunsaturated fatty acids which are released from the metabolic pathway of glycerol phospholipid degradation and are the main components of the phospholipid membrane. AA and DHA participate in membrane fluidity, signal transduction, and gene transcription during the whole life process (Rapoport et al., 2001). They are also involved in many pathological processes, including stroke (Rapoport, 2008). AA is stored in the phospholipid membranes of cells, which produce free AA by deacylation mediated by phospholipase A2 (PLA2). In pathological environments such as stroke, free AA increases the production of free radicals through the "arachidonic acid cascade reaction" (Rink and Khanna, 2011). This reaction can occur as early as $1 \mathrm{~h}$ after a stroke (Shohami et al., 1982), a major factor in the oxidative damage of tissues after a stroke. Consistent with the results of our study, previous studies reported a significant increase in the types of reactive oxygen species and AA metabolism after reperfusion in IS (Gürsoy-Ozdemir et al., 2004). 


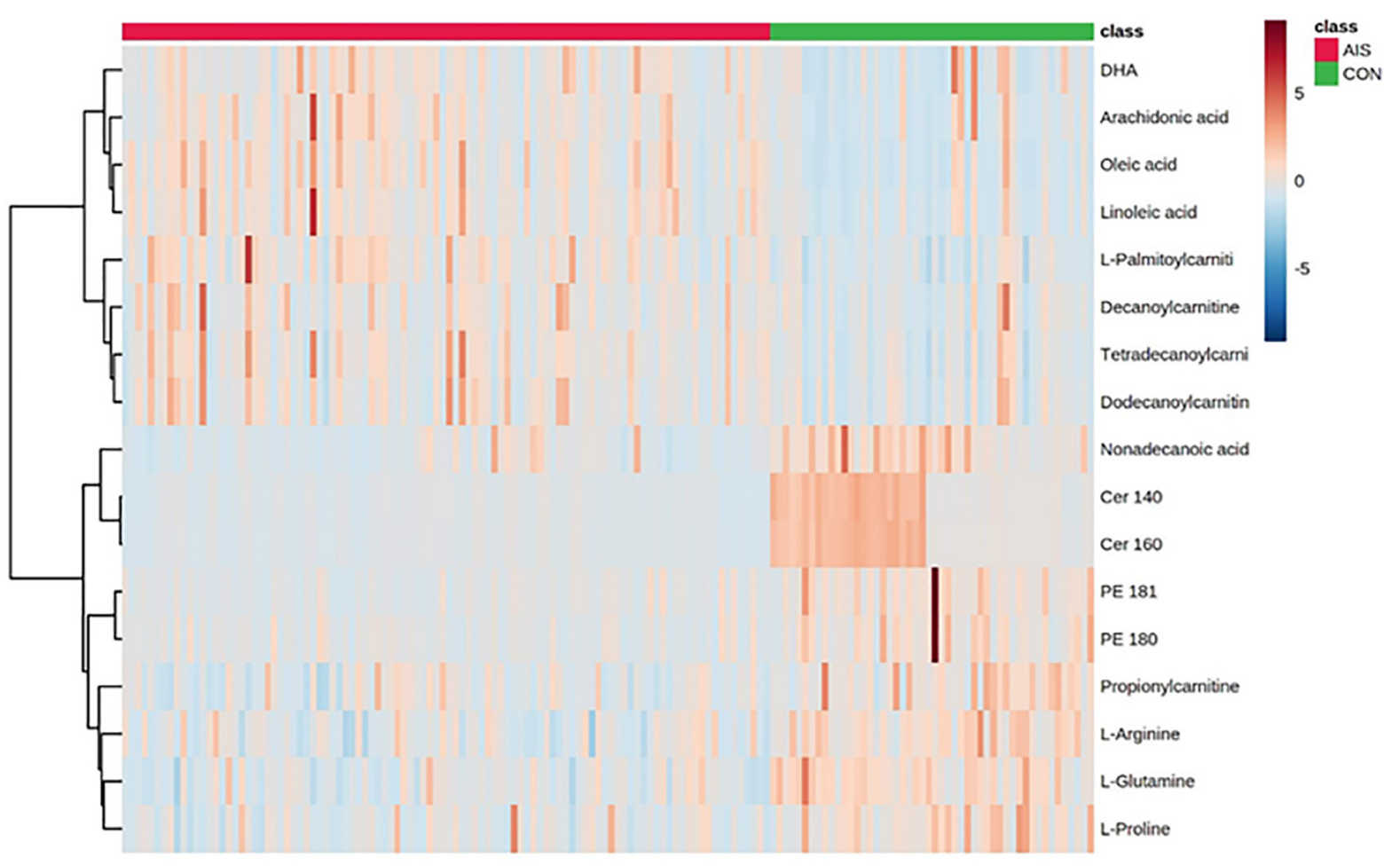

FIGURE 2 | Heat map of different serum metabolites between patients with acute ischemic stroke and healthy control groups.
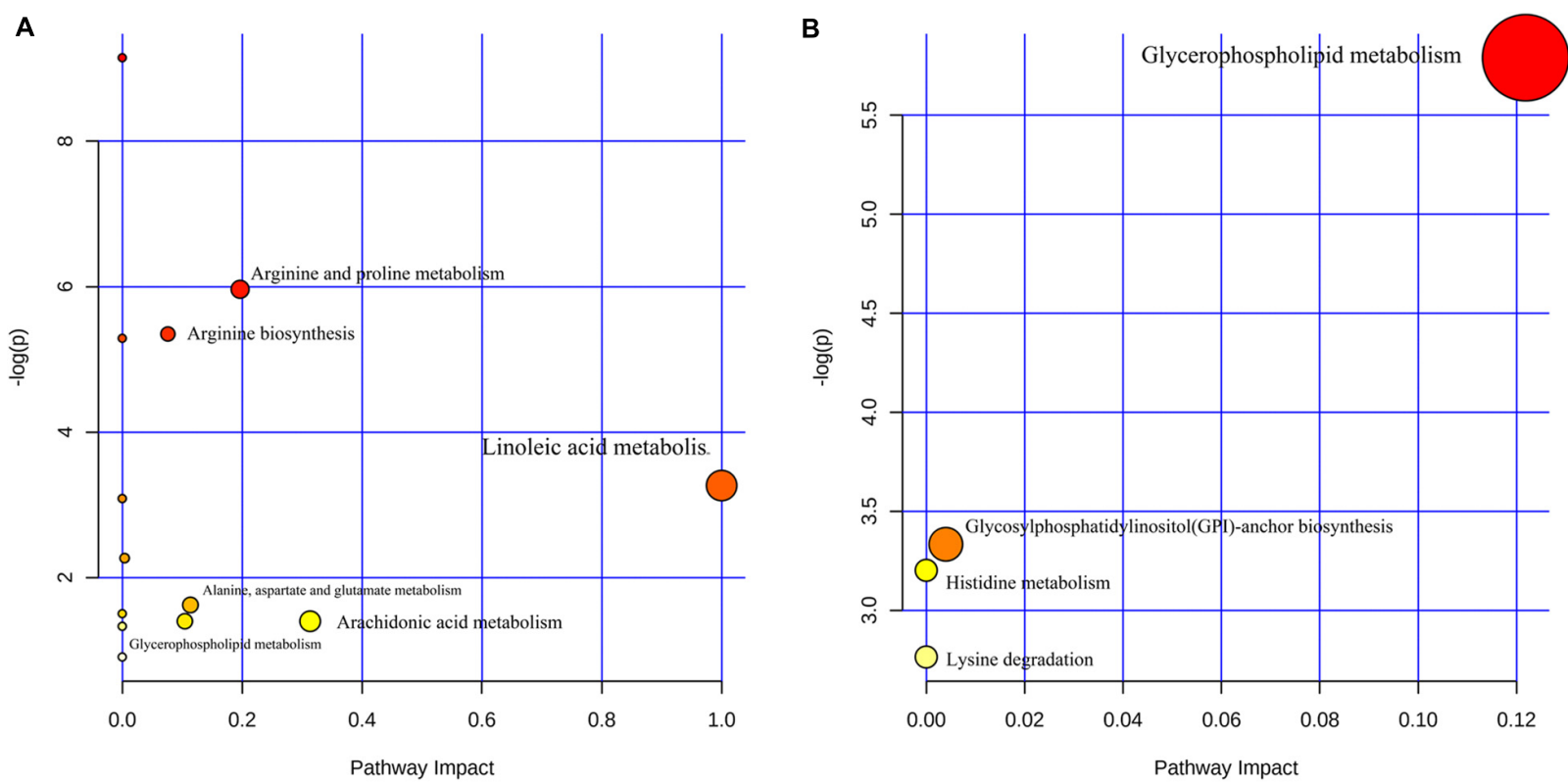

FIGURE 3 | Different metabolic pathways (A) between patients with acute ischemic stroke and healthy control groups and (B) between the large artery atherosclerosis and the small artery occlusion groups.

Ceramide is a type of waxy lipid composed of sphingosine and fatty acids, which plays a role in plaque formation (Holland and Summers, 2008). In addition, ceramide levels in the highrisk groups with IS were higher than those in the low-risk groups (Wang et al., 2017). However, previous studies reported that the level of ceramide in patients with AIS is lower than that in the HC group, which may be related to ceramide-mediated apoptosis (Taha et al., 2006). The specific mechanism warrants further study. Glutamine (Gln) and glutamate (Glu) can be converted into each other in the human body. The level of glutamine and 
the ratio of Gln to Glu were negatively correlated with the risk factors of cardiovascular disease (including body mass index, waist circumference, fasting blood glucose, insulin, triglyceride, etc.) (Cheng et al., 2012). Zheng et al. (2016) demonstrated that the ratio of Gln to Glu was associated with a reduced risk of cardiovascular disease. Similarly, glutamine levels in the AIS group were lower than those in the HC group of our study.

In the different metabolites of the LAA group and the SAO group, L-pipecolic acid mainly affects the lysine degradation pathway because lysine is produced in the process of L-pipecolic acid degradation, and lysine has previously been shown to decrease in patients with IS (Kimberly et al., 2013; Lee et al., 2017). 1-Methylhistidine is involved in histidine metabolism, which is a metabolic byproduct of the antioxidant molecule carnosine and its analogs in the brain (Bellia et al., 2011). $\mathrm{Hu}$ et al. (2019) showed that the level of L-pipecolic acid in patients with post-stroke depression is lower than that in HCs but higher than that in patients with stroke. PE, LysoPC, and LysoPE participate in glycerophospholipid metabolism and GPIanchor biosynthesis. LysoPE is a product of PE hydrolyzed by PLA2, which plays a role in cell-mediated cell signaling and the activation of other enzymes (Park et al., 2007). PE and LysoPC are intermediate products of glycerophospholipid metabolism, while AA and DHA are glycerophospholipid degradation products. It should be noted that the metabolic changes of glycerophospholipids can not only help diagnose AIS but also help distinguish different subtypes of IS. This might shed insight on our exploration of the pathological mechanisms of different subtypes. Studies have confirmed the correlation between lipid metabolites and AIS using lipidomic and metabolomics techniques (Yang et al., 2017; Au, 2018). Our results are consistent with these (Liu et al., 2017). To date, there has been no research exploring the mechanisms of different metabolite isomers showing different behaviors. This may require further lipidomic assessments in a larger sample size and further verification in animal models.

In this study, non-targeted metabolomics based on LC-MS was used to identify the different metabolites between patients with AIS and the HCs and between the LAA and SAO groups, providing new insights and encouraging further study of the pathophysiological mechanisms among different subtypes of IS. However, this study still has many limitations. First, this was a single-center study with a relatively small sample size. Multicenter studies with larger sample sizes will be needed to validate our findings. In addition, because the metabolites in the human body change dynamically, we collected the serum after the onset of the disease, which may have affected the estimation of the correlation between the metabolic differences and the disease. A longitudinal comparison of multiple blood samples, after the onset, from the same patient will enable a clearer assessment

\section{REFERENCES}

Adams, H. P., Bendixen, B. H., Kappelle, L. J., Biller, J., Love, B. B., Gordon, D. L., et al. (1993). Classification of subtype of acute ischemic stroke. Definitions for use in a multicenter clinical trial. TOAST. Trial of Org 10172 in acute stroke treatment. Stroke 24, 35-41. doi: 10.1161/01.str. 24.1 .35 of the changes in serum metabolites in AIS patients. Finally, targeted metabolomics technology in another set of samples is needed to further verify the different metabolites. In the future, to validate the results and investigate the potential of metabolites as biomarkers, we will include patients and follow them up prospectively to obtain their modified Rankin Scale scores and further explore metabolites associated with prognosis.

\section{CONCLUSION}

In summary, this study identified the different metabolites and metabolic pathways in patients with AIS and HCs and between the LAA and SAO subtypes of IS by non-targeted metabolomics. We demonstrated that metabolomics might be used to diagnose AIS and distinguish its subtypes. Further research is needed to explore the pathophysiological mechanisms that affect the changes in metabolites and lead to new clinical diagnoses and potential interventions.

\section{DATA AVAILABILITY STATEMENT}

The raw data supporting the conclusions of this article will be made available by the authors, without undue reservation.

\section{ETHICS STATEMENT}

The studies involving human participants were reviewed and approved by The First Affiliated Hospital of Zhengzhou University. The patients/participants provided their written informed consent to participate in this study.

\section{AUTHOR CONTRIBUTIONS}

XW, BS, and J-SK conceived and designed the research. XW, MT, and LZ conducted the experiments. XW, MT, LZ, L-IP, WS, and JL performed the data collection. XW, XL, RZ, JW, and SS analyzed the data. XW wrote the manuscript. All authors have read and approved the manuscript.

\section{FUNDING}

This study was funded by the National Key Research and Development Program, Major Chronic Non-communicable Disease Prevention and Control Research Key Special Project (2017YFC1308202), and Henan Provincial Medical Science and Technology Research Plan (SBGJ2018031).

Au, A. (2018). Metabolomics and lipidomics of ischemic stroke. Adv. Clin. Chem. 85, 31-69. doi: 10.1016/bs.acc.2018.02.002

Belgardt, B. F., and Brüning, J. C. (2010). CNS leptin and insulin action in the control of energy homeostasis. Ann. N. Y. Acad. Sci. 1212, 97-113.

Bellia, F., Vecchio, G., Cuzzocrea, S., Calabrese, V., and Rizzarelli, E. (2011). Neuroprotective features of carnosine in oxidative driven diseases. Mol. Aspects Med. 32, 258-266. doi: 10.1016/j.mam.2011.10.009 
Benjamin, E. J., Blaha, M. J., Chiuve, S. E., Cushman, M., Das, S. R., Deo, R., et al. (2017). Heart disease and stroke statistics-2017 update: a report from the american heart association. Circulation 135, e146-e603. doi: 10.1161/CIR. 0000000000000485

Cai, Y., Zhang, Y., Ke, X., Guo, Y., Yao, C., Tang, N., et al. (2019). Transcriptome sequencing unravels potential biomarkers at different stages of cerebral ischemic stroke. Front. Genet. 10:814. doi: 10.3389/fgene.2019.00814

Chamorro, Á, Meisel, A., Planas, A. M., Urra, X., van de Beek, D., and Veltkamp, R. (2012). The immunology of acute stroke. Nat. Rev. Neurol. 8, 401-410. doi: 10.1038/nrneurol.2012.98

Chen, P.-H., Gao, S., Wang, Y.-J., Xu, A.-D., Li, Y.-S., and Wang, D. (2012). Classifying ischemic stroke, from TOAST to CISS. CNS Neurosci. Therap. 18, 452-456. doi: 10.1111/j.1755-5949.2011.00292.x

Cheng, S., Rhee, E. P., Larson, M. G., Lewis, G. D., McCabe, E. L., Shen, D., et al. (2012). Metabolite profiling identifies pathways associated with metabolic risk in humans. Circulation 125, 2222-2231. doi: 10.1161/CIRCULATIONAHA. 111.067827

Ebert, D., Haller, R. G., and Walton, M. E. (2003). Energy contribution of octanoate to intact rat brain metabolism measured by $13 \mathrm{C}$ nuclear magnetic resonance spectroscopy. J. Neurosci. 23, 5928-5935. doi: 10.1523/jneurosci.23-13-05928. 2003

Fukuyama, N., Takizawa, S., Ishida, H., Hoshiai, K., Shinohara, Y., and Nakazawa, H. (1998). Peroxynitrite formation in focal cerebral ischemia-reperfusion in rats occurs predominantly in the peri-infarct region. J. Cereb. Blood Flow Metab. 18, 123-129. doi: 10.1097/00004647-199802000-00001

Gürsoy-Ozdemir, Y., Can, A., and Dalkara, T. (2004). Reperfusion-induced oxidative/nitrative injury to neurovascular unit after focal cerebral ischemia. Stroke 35, 1449-1453. doi: 10.1161/01.str.0000126044.83777.f4

Holland, W. L., and Summers, S. A. (2008). Sphingolipids, insulin resistance, and metabolic disease: new insights from in vivo manipulation of sphingolipid metabolism. Endocr. Rev. 29, 381-402. doi: 10.1210/er.2007-2025

Hu, Z., Fan, S., Liu, M., Zhong, J., Cao, D., Zheng, P., et al. (2019). Objective diagnosis of post-stroke depression using NMR-based plasma metabonomics. Neuropsychiatr. Dis. Treat. 15, 867-881. doi: 10.2147/NDT.S192307

Jickling, G. C., and Sharp, F. R. (2015). Biomarker panels in ischemic stroke. Stroke 46, 915-920. doi: 10.1161/STROKEAHA.114.005604

Jung, J. Y., Lee, H.-S., Kang, D.-G., Kim, N. S., Cha, M. H., Bang, O.-S., et al. (2011). 1H-NMR-based metabolomics study of cerebral infarction. Stroke 42, 1282-1288. doi: 10.1161/STROKEAHA.110.598789

Kelly, P. J., Albers, G. W., Chatzikonstantinou, A., De Marchis, G. M., Ferrari, J., George, P., et al. (2016). Validation and comparison of imaging-based scores for prediction of early stroke risk after transient Ischaemic attack: a pooled analysis of individual-patient data from cohort studies. Lancet Neurol. 15, 1238-1247. doi: 10.1016/S1474-4422(16)30236-30238

Kimberly, W. T., Wang, Y., Pham, L., Furie, K. L., and Gerszten, R. E. (2013). Metabolite profiling identifies a branched chain amino acid signature in acute cardioembolic stroke. Stroke 44, 1389-1395. doi: 10.1161/STROKEAHA.111. 000397

Lai, T. W., Zhang, S., and Wang, Y. T. (2014). Excitotoxicity and stroke: identifying novel targets for neuroprotection. Prog. Neurobiol. 115, 157-188. doi: 10.1016/ j.pneurobio.2013.11.006

Latchaw, R. E., Alberts, M. J., Lev, M. H., Connors, J. J., Harbaugh, R. E., Higashida, R. T., et al. (2009). Recommendations for imaging of acute ischemic stroke: a scientific statement from the American heart association. Stroke 40, 3646-3678. doi: 10.1161/STROKEAHA.108.192616

Lee, Y., Khan, A., Hong, S., Jee, S. H., and Park, Y. H. (2017). A metabolomic study on high-risk stroke patients determines low levels of serum lysine metabolites: a retrospective cohort study. Mol. Biosyst. 13, 1109-1120. doi: $10.1039 / \mathrm{c} 6 \mathrm{mb} 00732 \mathrm{e}$

Li, Y., Mao, L., Gao, Y., Baral, S., Zhou, Y., and Hu, B. (2015). MicroRNA-107 contributes to post-stroke angiogenesis by targeting Dicer-1. Sci. Rep. 5:13316. doi: 10.1038/srep13316

Liu, P., Li, R., Antonov, A. A., Wang, L., Li, W., Hua, Y., et al. (2017). Discovery of metabolite biomarkers for acute ischemic stroke progression. J. Proteome Res. 16, 773-779. doi: 10.1021/acs.jproteome.6b00779

Montaner, J., Perea-Gainza, M., Delgado, P., Ribó, M., Chacón, P., Rosell, A., et al. (2008). Etiologic diagnosis of ischemic stroke subtypes with plasma biomarkers. Stroke 39, 2280-2287. doi: 10.1161/STROKEAHA.107.505354
Panov, A., Orynbayeva, Z., Vavilin, V., and Lyakhovich, V. (2014). Fatty acids in energy metabolism of the central nervous system. Biomed. Res. Intern. 2014:472459. doi: 10.1155/2014/472459

Park, K. S., Lee, H. Y., Lee, S. Y., Kim, M.-K., Kim, S. D., Kim, J. M., et al. (2007). Lysophosphatidylethanolamine stimulates chemotactic migration and cellular invasion in SK-OV3 human ovarian cancer cells: involvement of pertussis toxin-sensitive G-protein coupled receptor. FEBS Lett. 581, 4411-4416. doi: 10.1016/j.febslet.2007.08.014

Rapoport, S. I. (2008). Arachidonic acid and the brain. J. Nutr. 138, 25152520.

Rapoport, S. I., Chang, M. C., and Spector, A. A. (2001). Delivery and turnover of plasma-derived essential PUFAs in mammalian brain. J. Lipid Res. 42, 678-685.

Rink, C., and Khanna, S. (2011). Significance of brain tissue oxygenation and the arachidonic acid cascade in stroke. Antioxid. Redox Signal. 14, 1889-1903. doi: 10.1089/ars.2010.3474

Schwartz, M. W., Woods, S. C., Porte, D., Seeley, R. J., and Baskin, D. G. (2000). Central nervous system control of food intake. Nature 404, 661-671. doi: $10.1038 / 35007534$

Shohami, E., Rosenthal, J., and Lavy, S. (1982). The effect of incomplete cerebral ischemia on prostaglandin levels in rat brain. Stroke 13, 494-499. doi: 10.1161/ 01.str.13.4.494

Song, B., Fang, H., Zhao, L., Gao, Y., Tan, S., Lu, J., et al. (2013). Validation of the ABCD3-I score to predict stroke risk after transient ischemic attack. Stroke 44, 1244-1248. doi: 10.1161/STROKEAHA.113.000969

Taha, T. A., Mullen, T. D., and Obeid, L. M. (2006). A house divided: ceramide, sphingosine, and sphingosine-1-phosphate in programmed cell death. Biochim. Biophys. Acta 1758, 2027-2036. doi: 10.1016/j.bbamem.2006.10.018

Tobe, E. H. (2013). Mitochondrial dysfunction, oxidative stress, and major depressive disorder. Neuropsychiatr. Dis. Treat. 9, 567-573. doi: 10.2147/NDT. S44282

Wang, D. D., Toledo, E., Hruby, A., Rosner, B. A., Willett, W. C., Sun, Q., et al. (2017). Plasma ceramides, mediterranean diet, and incident cardiovascular disease in the PREDIMED trial (Prevención con Dieta Mediterránea). Circulation 135, 2028-2040. doi: 10.1161/CIRCULATIONAHA.116.024261

Wang, H., Liddell, C. A., Coates, M. M., Mooney, M. D., Levitz, C. E., Schumacher, A. E., et al. (2014). Global, regional, and national levels of neonatal, infant, and under-5 mortality during 1990-2013: a systematic analysis for the Global Burden of Disease Study 2013. Lancet 384, 957-979. doi: 10.1016/S01406736(14)60497-60499

Wang, X., Tian, X., Pei, L.-L., Niu, P.-P., Guo, Y., Hu, R., et al. (2020). The association between serum Apelin-13 and the prognosis of acute ischemic stroke. Transl. Stroke Res. 11, 700-707. doi: 10.1007/s12975-019-00769-w

World Health Organization [WHO] (1989). Stroke-1989. Recommendations on stroke prevention, diagnosis, and therapy. Report of the WHO task force on stroke and other cerebrovascular disorders. Stroke 20, 1407-1431. doi: 10.1161/ 01.str.20.10.1407

Yang, L., Lv, P., Ai, W., Li, L., Shen, S., Nie, H., et al. (2017). Lipidomic analysis of plasma in patients with lacunar infarction using normal-phase/reversedphase two-dimensional liquid chromatography-quadrupole time-of-flight mass spectrometry. Anal. Bioanal. Chem. 409, 3211-3222. doi: 10.1007/s00216-0170261-266

Zheng, Y., Hu, F. B., Ruiz-Canela, M., Clish, C. B., Dennis, C., Salas-Salvado, J., et al. (2016). Metabolites of glutamate metabolism are associated with incident cardiovascular events in the PREDIMED PREvención con DIeta MEDiterránea (PREDIMED) Trial. J. Am. Heart Assoc. 5:e003755. doi: 10.1161/JAHA.116. 003755

Conflict of Interest: The authors declare that the research was conducted in the absence of any commercial or financial relationships that could be construed as a potential conflict of interest.

Copyright (c) 2021 Wang, Zhang, Sun, Pei, Tian, Liang, Liu, Zhang, Fang, Wu, Sun, Xu, Kang and Song. This is an open-access article distributed under the terms of the Creative Commons Attribution License (CC BY). The use, distribution or reproduction in other forums is permitted, provided the original author(s) and the copyright owner(s) are credited and that the original publication in this journal is cited, in accordance with accepted academic practice. No use, distribution or reproduction is permitted which does not comply with these terms. 